\title{
EFECTO DEL USO DE ADITIVOS EN DIETAS DE CODORNICES REPRODUCTORES (Coturnix coturnix japonica) BAJO CONDICIONES DE VERANO EN LA COSTA CENTRAL
}

\section{EFFECT OF THE USE OF ADDITIVES IN QUAIL BREEDERS (Coturnix coturnix japonica) DIETS UNDER CONDITIONS OF CENTRAL COAST SUMMERTIME}

\author{
${ }^{1}$ Pedro Ciriaco Castañeda y ${ }^{2}$ Henry David Roncal Ñañez
}

\begin{abstract}
Resumen
Se evaluaron los efectos de la adición en la dieta de $0.05 \%$ del Complejo B, $0.05 \%$ de Vitamina C, $0.2 \%$ de Bicarbonato de Sodio y $0.05 \%$ de Ácido Acetil Salicílico, sobre los parámetros productivos y reproductivos de codornices japonesas en época de verano en la costa central del Perú (Lima), con temperatura ambiental promedio de $25^{\circ} \mathrm{C}$. Codornices reproductores (270 hembras y 90 machos) de 48 días de edad, fueron distribuidas en cinco tratamientos (4 con aditivos y una dieta control) con tres repeticiones cada una, en un diseño estadístico completamente al azar. La suplementación con los aditivos no mejoró estadísticamente los parámetros productivos y reproductivos, observándose una tendencia positiva en la fertilidad, incubabilidad y natalidad. La respuesta, probablemente se debieron a la capacidad de adaptación de las codornices a las condiciones del verano, la cual no generó una necesidad de aditivos que contrarresten los efectos del calor. El uso de bicarbonato de sodio proporcionó la mayor retribución económica.
\end{abstract}

Palabras clave: Codorniz, Calor, Complejo B, Vitamina C, Bicarbonato de Sodio, Ácido Acetil Salicílico, Producción, Reproducción,

\begin{abstract}
The effect of dietary supplementation of $0.05 \%$ B complex, $0.05 \%$ Vitamin C, $0.2 \%$ Sodium bicarbonate and $0.05 \%$ Acetylsalicylic acid on productive and reproductive parameters of Japanese quail was evaluated in the summertime in the central coast of Peru (Lima), with an average temperature of $25^{\circ} \mathrm{C}$. Forty eight days old breeder quails (270 females and 90 males), were randomly distributed in five treatmentsin a statistical random design. No statistical differences for the parameters evaluated were found.Additives did not improve the productive and reproductive parameters but there was a positive effect on fertility, egg incubation and hatchability. These results, probably due to the adaptability of quail summer conditions, which did not generate a need to use additives that counteract the effects of heat. The use of sodium bicarbonate provided the best economic retribution
\end{abstract}

Keys words: Quail, Heat, B complex, Vitamin C, Sodium bicarbonate, Acetylsalicylic acid, Production, Reproduction.

\section{Introducción}

La crianza de codornices (Coturnix coturnix japónica) es una actividad avícola de alto rendimiento. La baja inversión inicial, los pocos requerimientos de espacios de terreno, el rápido retorno de capital, el valor nutricional de su carne y huevos, bajo costo de producción, alto nivel productivo; así como su rápido crecimiento, rusticidad y la precocidad de esta especie, son algunas de las características que la hacen atractiva para la producción y la convierte en una fuente alternativa de nutrientes a través del huevo.

Para el proceso productivo y reproductivo, los investigadores han establecido temperaturas favorables para el desempeño productivo y reproductivo adecuado de las codornices entre los $18 \mathrm{a} 21^{\circ} \mathrm{C}$ y la humedad relativa entre 60 y $80 \%$, con variaciones no muy pronunciadas indicando que los problemas del estrés por calor pueden ocurrir por encima de $\operatorname{los} 27^{\circ} \mathrm{C}$, sobre todo en los meses de verano cuando el ave está expuesta a temperaturas por encima de la zona de su confort térmico, siendo frecuente apreciar una disminución en su producción y productividad (Lima, 1996; Murakami y Ariki, 1998).

En épocas de calor, las aves empiezan a jadear y disipan el calor corporal al medio ambiente a través de radiación, conducción, convección y evaporación. El ave adopta posiciones para aumentar el área de la superficie vascular

\footnotetext{
${ }^{1}$ Facultad Zootecnia, Dpto. de Produccion Animal de la UNALM E-mail: pciriaco@lamolina.edu.pe

${ }^{2}$ Ingeniero Zootecnista
} 
por vasodilatación, incrementando el consumo de agua y una aceleración del ritmo respiratorio. También, estiran sus extremidades y alas, lo que le permite una mayor circulación de aire por su cuerpo y disminuye el consumo de alimento buscando equilibrar su termólisis (Díaz, 2009). Si la temperatura continúa incrementándose, será incapaz de desarrollar sus mecanismos de defensa y puede morir porque la posibilidad de perder calor por medios no evaporativos (la perdida de calor a través de la piel), se reduce notablemente, conduciendo a la pérdida del equilibrio ácido-básico acentuado por una hipoxia crónica, la cual afectará también el crecimiento, eficacia alimenticia, viabilidad, calidad de la cáscara del huevo, y la reproducción (Chauca, 2001 y Nilipour, 2003). A temperaturas superiores a $30^{\circ} \mathrm{C}$, la mortalidad puede superar e1 $20 \%$, disminuyendo el peso de ovarios y número de folículos largos (Rozenboimetal., 2007).

Para enfrentar al calor se han utilizado el complejo B y la vitamina $\mathrm{C}$. El primero, funciona como coenzimas y catalizadores de reacciones químicas que ocurren a nivel celular, y metabólicas que liberan la energía contenida en los alimentos, además participan en el control de procesos de síntesis de células sanguíneas (hematopoyéticas), coagulación de la sangre y la respiración celular (Bender, 1998). Bajo condiciones de hipotermia, Sahin etal. (2003), utilizando $1 \mathrm{mg} / \mathrm{kg}$ de alimento de complejo $\mathrm{B}$ en dietas de codornices, mejoraron los parámetros productivos en la etapa de postura. Por otro lado, la vitamina $C$, participa en la síntesis de corticosteroides que estimula los mecanismos de defensa como la actividad fagocítica, la formación de anticuerpos, disminuye la deshidratación celular antes y durante el estrés por calor, mejorando el balance de iones potasio y sodio del plasma que están asociados con diarreas inducidas por las altas temperaturas (Inchem, 2006 y Weber, 2009).

Creel etal., (2006), al adicionar en el alimento $75 \mathrm{mg}$ de vitamina $\mathrm{C} / \mathrm{Kg}$ no encontraron mejoras en la producción, peso de huevo, calidad de cáscara, fertilidad y natalidad. Asimismo, Usman etal., (2008), no encontraron respuesta positiva a la adición de $0.1 \%$ de vitamina $\mathrm{C}$ vía alimento. Sin embargo, Yigit etal. (2002), concluyeron que la suplementación de $200 \mathrm{mg} / \mathrm{Kg}$ de alimento en gallinas, protege la vitamina A de la oxidación en el alimento y por lo tanto se observa incrementos de vitamina A y C séricas. El suministro de 500ppm de ácido ascórbico en la dieta de codornices, mejora el balance electrolítico de $\mathrm{K}^{+}$y $\mathrm{Na}^{+}$del plasma que están asociados con diarreas inducidas por la alta temperatura. Asimismo, mejoró la postura, peso del huevo, el grosor de la cáscara del huevo (Bardakcio etal., 2004, Önol etal., 2005).

Bajo condiciones de calor, la adición de Bicarbonato de sodio de $1 \mathrm{Kg} / \mathrm{TM}$ de alimento, es un mejorador del balance electrolítico ( $\mathrm{Na}+, \mathrm{K}-, \mathrm{Cl}-\mathrm{meq} / \mathrm{Kg})$ en la dieta, que influye positivamente en la calidad de la cáscara, peso corporal y reduce la mortalidad, evita la acidosis metabólica, mejora la digestibilidad proteica, la conversión de alimentos y el rendimiento ya que la mayoría de las rutas metabólicas funcionan en las condiciones óptimas requeridas dirigiéndose al proceso de crecimiento en lugar de ir a la regulación homeostática (Kaplan etal., 2005; Lesson, 2010). Asimismo, adicionando $2 \%$ de bicarbonato de sodio, en dietas de codornices se obtuvo mayores porcentajes de postura, masa de huevos, eficiencia alimenticia y retribución económica(Flores 2008)

El ácido acetilsalicílico (AAS), es un ácido orgánico antiinflamatorio no esteroideo de la familia de los salicilatos y es definido como un producto metabólico de la aspirina (Katzung, 2007). Mc Danieletal., (1993) no encontraron respuesta positiva en la calidad de cáscara, conversión alimenticia al adicionar AAS a la dieta de reproductoras pesadas. Pero, bajo condiciones de hipertermia, Galil (2004), obtuvo mejor producción y masa de huevos. Asimismo, Mohammed (2010) observó que la incorporación de AAS en dietas de gallinas expuestas a temperaturas de $33^{\circ} \mathrm{C}$ a $39^{\circ} \mathrm{C}$, mejoró la producción de huevos, peso de huevo, grosor de cáscara. Asimismo, codornices criadas a temperaturas de 34 a $40^{\circ} \mathrm{C}$, fueron alimentadas con dietas suplementadas con 0.05 y $0.1 \%$ de AAS, encontrándose mayor producción de huevos, fertilidad, y eclosión, además de una menor mortalidad embrionaria (Hassan etal., 2003; Abou ElSoud etal., 2006).

Las codornices ingieren entre 20 y 25 g por ave por día, siendo el porcentaje de postura un promedio de $80 \%$ y variando el peso del huevo de 9 a12 g. La conversión alimenticia varía entre 2.6 y a 3.5. El porcentaje de porcentaje de mortalidad en los reproductores debe considerarse entre 3 a 5 \% (Cabrejos, 2008, Flores, 2008). El porcentaje de fertilidad de 70 a $90 \%$ y los porcentajes de incubabilidad y natalidad de 80 a $84 \%$ y 60 a 70\%, respectivamente, fueron reportados por Galindez etal. (2009).

La costa central del Perú está comprendida desde el litoral marino hasta el piso inferior andino de los departamentos de Ancash, Lima e Ica. Las temperaturas máximas y mínimas en estas localidades costeras están condicionadas por la temperatura superficial del agua de mar por la incursión de masas de aire cálido del norte o por la intensificación de los vientos del sur, los cuales por lo general desmejoran el tiempo en la costa. Las temperaturas más altas ocurren en el mes de febrero, alcanzando un valor promedio mensual para las máximas y mínimas de $27^{\circ} \mathrm{C}$ y $20^{\circ} \mathrm{C}$ y las temperaturas más bajas se presentan en el mes de agosto, siendo las máximas de $19^{\circ} \mathrm{C}-20^{\circ} \mathrm{C}$, y las mínimas $14^{\circ} \mathrm{C}-15^{\circ} \mathrm{C}$. Durante periodos del fenómeno del niño, se presenta un incremento de las temperaturas diurnas y nocturnas, alcanzando los $31.4^{\circ} \mathrm{C}$ y las mínimas $\operatorname{los} 25^{\circ} \mathrm{C}$, (Cuba e Ita, 2010).

La crianza de las codornices mayormente se desarrolla en la costa central, por lo tanto, para reducir los posibles efectos negativos del calor en el verano, se pueden utilizar aditivos en la dieta, existentes en nuestro medio, los cuales van actuar de modo diferente en el organismo animal, ya sea activando mecanismos fisiológicos que 
reducen las pérdidas por efecto del calor y/o optimizando el aprovechamiento de los nutrientes contenidos en el alimento. Por ello, el objetivo de la presente investigación, es cuantificar los rendimientos productivos y reproductivos al incluir como aditivos al complejo $\mathrm{B}$, la vitamina $\mathrm{C}$, el bicarbonato de sodio y el ácido acetilsalicílico en dietas de codornices reproductoras bajo condiciones de verano de la costa central del Perú, indicando la rentabilidad de su uso.

\section{Materiales y métodos}

La investigación se realizó en la Unidad Experimental de Avicultura de la Universidad Nacional Agraria La Molina con una duración de 3 meses entre Enero a Marzo, con temperatura promedio máxima y mínima de $27^{\circ} \mathrm{c}$ y $24^{\circ} \mathrm{C}$, respectivamente. Se utilizaron 30 jaulas metálicas equipadas de $60 \mathrm{~cm}$ de largo, $40 \mathrm{~cm}$ ancho y $17 \mathrm{~cm}$ de alto, en un galpón de $6 \mathrm{~m}$ de ancho, $16 \mathrm{~m}$ largo y con una altura de $3 \mathrm{~m}$. Se usó una mezcladora horizontal de $400 \mathrm{Kg}$ para uniformizar los aditivos y una incubadora eléctrica para el desarrollo de los huevos fértiles.

Los productos evaluados fueron: El Complejo B en polvo, la vitamina $\mathrm{C}$ en polvo de apariencia de cristales blanquecinos transparentes, con un grado de pureza del $99 \%$, el bicarbonato de sodio, con un grado de pureza del 99.74\% y en polvo color blanco y el ácido acetil salicílico con $100 \%$ de pureza, de apariencia polvo blanquecina.

Se utilizó codornices de 48 días de edad (270 hembras y 90 machos con proporción de 1 macho para 3 hembras). Se mantuvo las reglas de bioseguridad preestablecidas de la Unidad. Para la evaluación estadística de los parámetros se utilizó el diseño completamente al Azar (DCA), con cinco tratamientos y tres repeticiones (Calzada, 1982).

Se preparó una dieta basal, Según las recomendaciones del N.R.C (1994), a la cual se le adicionaron los productos.

Los tratamientos fueron:

T1: Dieta Basal (control)

T2: Dieta Basal $+0.05 \%$ de Complejo B

T3: Dieta Basal $+0.05 \%$ de Vitamina C

T4: Dieta Basal $+0.2 \%$ de Bicarbonato de Sodio

T5: Dieta Basal $+0.05 \%$ de Ácido Acetil Salicílico

Se evaluaron los siguientes parámetros productivos reproductivos: Numero de huevos, porcentaje de postura, peso promedio del huevo, masa de huevo, consumo de alimento, conversión alimenticia, mortalidad, porcentaje de fertilidad, incubabilidad y natalidad. Asimismo, se determinó la retribución económica, por las ganancias obtenidas en número de huevos fértiles producidos y número de cotupollos nacidos.

\section{Resultados y discusión}

En el Tabla 1, se indica que no existen diferencias significativas entre los tratamientospara todos los parámetros productivos. Los aditivos utilizados en la dieta no tuvieron un efecto positivo en comparación con la dieta control.

Tabla 1. Efecto de los aditivos sobre los Parámetros Productivos.

\begin{tabular}{lccccc}
\hline \multicolumn{1}{c}{ Parámetros } & T-1 & T-2 & T-3 & T-4 & T-5 \\
\hline Total Huevos acumulados & 3,621 & 3,558 & 3,582 & 3,661 & 3,558 \\
Porcentaje de Postura & 79.83 & 78.44 & 78.97 & 80.71 & 78.44 \\
Peso Promedio Huevos (g) & 11.01 & 11.08 & 10.96 & 10.82 & 10.86 \\
Masa de Huevos (g) & 878.9 & 867.7 & 864.8 & 874.8 & 851.1
\end{tabular}

Consumo Alimento (g/ave/d) 20.9720 .3520 .0319 .9419 .88

$\begin{array}{llllll}\text { Conversión Alimenticia } & 3.67 & 3.61 & 3.56 & 3.54 & 3.58\end{array}$

T-1=Dieta basal; T-2 $=0.05 \%$ Complejo b; T-3 $=0.05 \%$ Vitamina $C$; T-4 $=0.2 \%$ Bicarbonato de Sodio; T-5 $=0.05 \%$ Ácido Acetilsalicílico

Las vitaminas presentes en el alimento, sobre todo aquellas involucradas en la síntesis de proteína (niacina, piridoxina, ácido fólico, cianocobalamina), estuvieron en cantidades necesarias para cumplir sus funciones. Asimismo, no influyeron en los estímulos sensoriales que generan impulsos nerviosos para dar una respuesta al consumo alimento o a una mayor ingesta de agua que permite indirectamente a elevar el consumo o un mejor aprovechamiento de los nutrientes que podría mejorar la eficiencia alimenticia como lo indica Weber (2009).

La temperatura máxima de $27^{\circ} \mathrm{C}$ a que estaban sometidas las codornices por algunos momentos no influyó en un contundente estrésque concuerda con Creel etal., (2001), quienes indican que las aves pueden acostumbrase a las altas temperaturas ocasionales. Sin embargo, no concuerda con Bardakcio etal., (2004), y Önol etal., (2005), quienes encontraron efectos positivos en parámetros productivos de codornices, pero sometidos a temperaturas constantes mayores de $30^{\circ} \mathrm{C}$. El proceso bioquímico del complejo $\mathrm{B}$, continuó con normalidad para la degradación de los nutrientes a temperaturas promedios de $25^{\circ} \mathrm{C}$. La adición de vitamina $\mathrm{C}$ no es necesaria para codornices bajo condiciones de manejo y ambientes normales, debido a que éstas pueden sintetizar ácido ascórbico. Probablemente, las respuestas más contundentes de la acción de las vitaminas se pueda observaren ambientes de altas temperaturas donde existe un efecto directo en la deshidratación corporal y celular en donde las vitaminas actúan para contrarrestar estos efectos donde posiblemente las vitaminas promueven un mejor balance de iones de sodio y potasio que permite un mejor transporte de los nutrientes y por ello puede observarse mejores respuestas frente al desafío que en condiciones normales. 
El tratamiento con bicarbonato de sodio, tampoco mejoró las respuestas productivas, concordando con Kaplanetal. (2005), probablemente porque las condiciones ambientales no provocaron una alcalosis respiratoria que induzca a un desequilibrio ácido-base para que exista una necesidad de bicarbonato de sodio. Sin embargo, no concuerda con Flores (2008), quien encontró diferencias al trabajar con una mayor dosis del bicarbonato, bajo las mismas condiciones de verano. Existe una tendencia hacia una mejora en el rendimiento productivo con la utilización del bicarbonato de sodio.

El tratamiento con Ácido acetilsalicílico, no resultó en efectos positivos en los parámetros evaluados, concordando con McDaniel etal., (1993), donde la adición de AAS no estuvo aunada a condiciones estresante de ambientes cálidos que puedan permitir la efectividad de este aditivo. Por otro lado, no concuerdacon la respuesta positiva al empleo de este aditivo hallado por Hassan etal., (2003), Galil (2004), Abou El-Soud etal., (2006) y Mohammed, (2010), donde al utilizar una mayor dosis en condiciones de altas temperaturas que el presente experimento, permitieron brindar un bienestar a las aves por sus efectos antipiréticos y analgésicos.Probablemente el nivel empleado en el presente experimento no afectó la producción de prostaglandinas en cantidades importantes para el mantenimiento de la mucosa intestinal que optimizan la absorción de nutrientes.Adicionalmente, no influyó en reducir los niveles de corticosterona los cuales hubieran generado el aumento de concentraciones de FSH y LH que activan los ovarios a producir más huevos. Es importante indicar que los problemas de disminución de la producción, se han presentado cuando las temperaturas están encima de $\operatorname{los} 30^{\circ} \mathrm{C}$, para el caso de este experimento, las temperaturas estuvieron alrededor de los $25^{\circ} \mathrm{C}$ por lo que puede inferirse que las codornices se adaptaron sin problema a las condiciones propias del verano de costa central peruana, además que probablemente el efecto estacional no es significativo sobre la producción

En el Tabla 2, se presentan los resultados del porcentaje de fertilidad, incubabilidad y natalidad. No se encontraron diferencias significativas entre los tratamientos para ninguno de los parámetros reproductivos evaluados.

Tabla 2. Efecto de los aditivos sobre los Parámetros Reproductivos.

\begin{tabular}{cccccc}
\hline Parametro & T-1 & T-2 & T-3 & T-4 & T-5 \\
\hline \% Fertilidad & 91.17 & 92.25 & 92.93 & 93.18 & 92.51 \\
\% Incubabilidad & 87.98 & 89.50 & 89.40 & 91.67 & 90.63 \\
\% Natalidad & 84.34 & 85.58 & 85.77 & 87.43 & 86.86 \\
\hline
\end{tabular}

T-1= Dieta basal; T-2=0.05\% Complejo B; T-3=0.05\% Vitamina C; T-4= $0.2 \%$ Bicarbonato de Sodio; T-5 $=0.05 \%$ Ácido Acetilsalicílico

La adición de las vitaminas no mejoró estadísticamente los parámetros reproductivos, concordando con Sahin etal. (2003) Usman etal., (2008) y Creel etal., (2006), sin embargo, no concuerda con Yigit etal. (2002). Bardakcio etal.(2004), Önol etal. (2005), quienes encontraron respuestas positivas en aves sometidas a temperaturas superiores al presente experimento. Por otro lado, se observó tendencia a una mejora en los rendimientos reproductivos con el uso de las vitaminas evaluadas, siendo superiores al grupo control. Probablemente, el complejo B generó una mejor asimilación de los ácidos grasos polinsaturados (que aportaba el aceite de palma de la dieta) importantes para el mantenimiento de las células sexuales en machos y hembras y las respuestas hormonales y nerviosas que permitieron la continuidad del líbido en los machos. Adicionalmente, las vitaminas actuaron a través de sus coenzimas corrigiendo la síntesis de aminoácidos. La vitamina $\mathrm{C}$ pudo haber mejorado la concentración espermática y redujo la peroxidación lipídica en las células espermáticas. Probablemente, se haya favorecido la concentración de antioxidantes en el huevo y por lo tanto los tejidos en formación de los cotupollos fueron más vigorosos para no verse afectados de las condiciones de incubación.

Los tratamientos con bicarbonato y ácido acetilsalicilico, tampoco mostraron diferencias estadísticas que concuerda con McDaniel etal., (1993), pero no concuerda con Hassan etal.(2003) y Abou El-Soud etal., (2006), quienes trabajaron bajo condiciones más altas de temperatura que el presente experimento.

Los tratamientos con bicarbonato de sodio y ácido acetilsalicílico, también tuvieron una tendencia hacia una mejor respuesta que el control.El bicarbonato de sodio pudo haber efectuado un mejor balance electrolítico promoviendo una mejor asimilación de los nutrientes del alimento que son necesarias para el mantenimiento de los procesos reproductivos como la ovulación y fertilización, influyendo en una mejor constitución de la cáscara del huevo que pudo permitir un correcta respiración del embrión en el proceso de incubación. El ácido acetilsalicílico, pudo haber promovido una disminución de las prostaglandinas y tromboxanos que permiten los efectos antipiréticos y antiinflamatorios para retornar en homeostasis a las codornices, influyendo en una mejor constitución de la cáscara de huevo que deriva en mejor protección del embrión y correcta respiración de los huevos en la incubadora. También, podría explicarse en la síntesis de defensas antioxidantes ferritínicas por la reproductoras para posteriormente transmitirlas al huevo y permitir a lo largo de los 16 días de incubación la viabilidad del embrión para su nacimiento.

No se observaron problemas de jadeos, heces sueltas, picaje, mortalidad súbita, malformaciones de cotupollos, que puedan haber indicado problemas de tolerancia a las variaciones de temperatura $\left(19^{\circ} \mathrm{C}-27^{\circ} \mathrm{C}\right)$ presentadas en el transcurso del experimento. Probablemente si se hubieran presentado mayores picos de temperatura y de forma constante, la adición de cualquiera de los aditivos podría haber tenido una respuesta significativa. 
En Tabla 3, se resumen los datos sobre la retribución económica por tratamiento, considerando el precio de venta por huevo fértil, por servicio de incubación y por cotupollo de S/. 0.25, S/. 100.00/un millar de huevos y $\mathrm{S} / .1 .0$, respectivamente y el costo de las dietas experimentales, todos correspondientesa los precios del mes de mayo del 2013. Se obtuvo una retribución económica en $1.23 \%$ mayor que el control en las respuestas productivas solo con la adición del bicarbonato de sodio. Sin embargo, se tuvo una mayor retribución económica al utilizar todos los aditivos en la obtención de cutopollos, siendo, el uso del bicarbonato de calcio el que proporcionó la mayor retribución económica.
La utilización de los aditivos: complejo B, vitamina C, bicarbonato de sodio y Ácido acetilsalicílico en la dieta de codornices en reproducción no mejoró los parámetros productivos y reproductivos. Sin embargo, generó una tendencia hacia una mejora solo en los parámetros reproductivos, obteniéndose los mas altos méritos económicos. Por lo que se recomienda que bajo condiciones de verano de costa central peruana, la utilización de los aditivos evaluados, especialmente el bicarbonato de sodio. Además, realizar evaluaciones productivas y reproductivas en codornices criadas en ambientes superiores de $27^{\circ} \mathrm{C}$.

Tabla 3. Retribución Económica por Producción de Huevo y por cotupollo.

\begin{tabular}{lccccc}
\hline Rubro & $\mathrm{T}-1$ & $\mathrm{~T}-2$ & $\mathrm{~T}-3$ & $\mathrm{~T}-4$ & $\mathrm{~T}-5$ \\
\hline Ingresos & & & & & \\
Total Huevos fértiles (unidades) & 3,589 & 3,523 & 3,547 & 3,593 & 3,537 \\
Precio por Huevo Fértil (S/.) & 0.25 & 0.25 & 0.25 & 0.25 & 0.25 \\
Ingresos por huevos (S/.) & $\mathbf{8 9 7 . 2 5}$ & $\mathbf{8 8 0 . 7 5}$ & $\mathbf{8 8 6 . 7 5}$ & $\mathbf{8 9 8 . 2 5}$ & $\mathbf{8 8 4 . 2 5}$ \\
Cotupollos nacidos (unidades) & 3,027 & 3,025 & 3,042 & 3,141 & 3,072 \\
Precio/cotupollo (S.) & 1.00 & 1.00 & 1.00 & 1.00 & 1.00 \\
Ingresos/cotupollo (S/.) & $\mathbf{3 , 0 2 7}$ & $\mathbf{3 , 0 2 5}$ & $\mathbf{3 , 0 4 2}$ & $\mathbf{3 , 1 4 1 . 0 0}$ & $\mathbf{3 , 0 7 2}$ \\
Egresos & & & & & \\
Consumo total de Alimento (Kg) & 144.90 & 140.70 & 138.50 & 137.80 & 137.40 \\
Costo/Kg. Alimento (S/.) & 1.22 & 1.23 & 1.25 & 1.23 & 1.28 \\
Total costo/alimentación(S/.) & $\mathbf{1 7 7 . 4 9}$ & $\mathbf{1 7 3 . 5 3}$ & $\mathbf{1 7 3 . 4 6}$ & $\mathbf{1 6 9 . 3 4}$ & $\mathbf{1 7 6 . 5 5}$ \\
Huevos incubados (unidades) & 3,589 & 3,523 & 3,547 & 3,593 & 3,537 \\
Precio incubación/huevo (S/.) & 0.10 & 0.10 & 0.10 & 0.10 & 0.10 \\
Total costo/incubación (S/.) & $\mathbf{3 5 8 . 9 0}$ & $\mathbf{3 5 2 . 3 0}$ & $\mathbf{3 5 4 . 7 0}$ & $\mathbf{3 5 9 . 3 0}$ & $\mathbf{3 5 3 . 7 0}$ \\
Retribución económica & & & & & \\
Por Huevo fértil & $\mathbf{7 1 9 . 7 6}$ & $\mathbf{7 0 7 . 2 2}$ & $\mathbf{7 1 3 . 2 9}$ & $\mathbf{7 2 8 . 9 1}$ & $\mathbf{7 0 7 . 7 0}$ \\
Relativo (\%) & $\mathbf{1 0 0}$ & $\mathbf{9 8 . 2 8}$ & $\mathbf{9 9 . 1 3}$ & $\mathbf{1 0 1 . 2 3}$ & $\mathbf{9 8 . 6 8}$ \\
Por cotupollo & $\mathbf{2 , 4 9 0 . 2}$ & $\mathbf{2 , 4 9 9 . 1 7}$ & $\mathbf{2 , 5 1 3 . 8 4}$ & $\mathbf{2 , 6 1 2 . 3 6}$ & $\mathbf{2 , 5 4 1 . 7 5}$ \\
Relativo (\%) & $\mathbf{1 0 0}$ & $\mathbf{1 0 0 . 4 3}$ & $\mathbf{1 0 1 . 5 1}$ & $\mathbf{1 0 6 . 2 1}$ & $\mathbf{1 0 3 . 0 2}$ \\
\hline
\end{tabular}

T-1= Dieta basal; T-2 $=0.05 \%$ Complejo B; T-3 $=0.05 \%$ Vitamina C; T-4= 0.2\% Bicarbonato de Sodio; T-5=0.05\% Ácido Acetilsalicílico. 


\section{Literatura citada}

Abou El-Soud, SB.; Ebeid, T. and Eid, YZ. 2006. Physiological and Antioxidative Effects of Dietary Acetyl Salicylic Acid in Laying Japanese Quail (Coturnixcoturnix japonica) under High Ambient Temperature. The Journal of Poultry Science. Vol. 43, No. 3 pp.255-265.

Bardakcio He, Glu., K. Seyreko, C. Yenisey, M. Serter, F. Kargin Kiral, and P. A. Ulutas. 2004. Effects of Dietary Vitamin C Supplementation on Some Serum Biochemical Parameters of Laying Japanese Quails Exposed To Heat Stress $\left(34.8^{\circ} \mathrm{C}\right)$.Revue Méd. Vét., 2004, 155, 6, 339-342

Bender, D. 1998. Nutritional Biochemistry of the Vitamins. New York: Cambridge University Press, 184222

Cabrejos, L. 2008. Evaluación de tres estándares de alimentación en la etapa de desarrollo de la codorniz japonesa (Coturnix Japónica) y su efecto en la etapa reproductiva. Tesis Ingeniero Zootecnista. Universidad Nacional Agraria La Molina, Facultad de Zootecnia. Lima, Perú.

Calzada, J. 1982. Métodos Estadísticos para la Investigación. Editorial Milagros S. A. Lima, Perú.

Chauca, D. 2001. Medio ambiente y Producción animal. Revista de investigaciones veterinarias Perú, suplemento 1: $37-42$

Creel, Lh.; Maurice, DV.; Lightsey, Sf and Grimes, LW. 2006. Stability of dietary ascorbic acid and the effect of supplementation on reproductive performance of broiler breeder chickens. Br Poult Sci. Mar;42(1):96-101.

Cuba, F. y N. Ita. 2010. Guía climática turística. Servicio Nacional de Meteorología e Hidrología del Perú. Impresión digital.

Diaz, G. 2009. http://www.scribd.com/doc/22891450/ Estres-Calorico-en-Aves Memorias\#logout.

Flores, P. 2008. Efecto del balance electrolítico de la dieta utilizando diferentes niveles de bicarbonato de sodio en el comportamiento productivo de la codorniz japonesa (Coturnix coturnix japónica). Tesis Ingeniero Zootecnista. Universidad Nacional Agraria La Molina, Facultad de Zootecnia. Lima, Perú.

Galil M. 2004. Effect of using some anti-heat stress compounds on the performance of some local breeds of chicken under hot climatic condition. EgyptianPoultryScienceJournal, Vol. 24, No. 2, 417-427

Galíndez, R.; G. M. Vasco de Basilio, Gonzalo Martínez, D. Vargas, E. Uztarizy P. Mejía. 2009. Evaluación de la fertilidad y eclosión en la codorniz japonesa. Zootecnia Trop., 27(1): 7-15.

Hassan et al., 2003. Effect of acetyl salicylic acid in drinking water on reproductive performance of Japanese quail (Coturnixcoturnix japonica). Poultry Science 82:1174-1180.
Inchem, 2006. www.inchem.org/documents/pims/ pharm/aspirin.htm\#PartTitle: 1.\%20NAME

Kaplan, O.; Avci, M.; and Yert, M. 2005. Effects of Ascorbic Acid on the Performance andSome Blood Parameters of Japanese Quails RearedUnder Hot Climate Conditions. Turk J VetAnimSci 29 (2005) 829-833

Katzung, B G. 2007. Farmacología básica y clínica. Editorial El Manual Moderno S.A. de C.V México ,1185pp

Lesson, S. 2010. Feeding programs for laying hens. 18th Annual ASAIM SE Asian Feed Technology and Nutrition Workshop. Le MeridienSiemReap, Cambodia.

Lima, M. E. P. 1996. Criação de codornas.CPT. Viçosa, MG. 44 p.

Mc Daniel, CD.; Balog, JM.; Freed, M.; Elkin, RG.; Wellenreiter, RH and Hester, PY. 1993. Response of layer breeders to dietary acetylsalicylic acid. 1. Effects on hen performance and eggshell quality. Poultry Science, $72: 1084-1092$

Mohammed, Abdullah A. 2010. Effect of Acetyl Salicylic Acid (ASA) in Drinking Water on Productive Performance and Blood Characteristic of Layer Hens During Heat Stress. International Journal of Poultry Science 9 (4): 382-385.

Murakami, A.E. y J. Ariki. 1998. Produção de codornas japonesas. $1^{\mathrm{a}} \mathrm{ed}$. FUNEP. Jaboticabal. $79 \mathrm{p}$.

Nilipour, A. H. 2003. Manejo en crianza y postura comerciales en estrés calóricos Notas personales.

National Research Cournal (NRC). 1994. Nutrient Requirements of Poultry rev Edic. National Academy Press, Washington, D.C.,U.S.A.

ÖNOL, AG.; HE. BARDAKÇIOĞLU; MK. TÜRKYILMAZ and A. NAZLIGÜL. 2005. Effects of Vitamin C Supplementation on Egg Production Traits and Eggshell Quality in Japanese Quails (Coturnixcoturnix japonica) Reared under High Ambient Temperature. Turk J Vet AnimSci29 1185-1189.

Rozenboim, M., Tako E., O. Gal-Garber; J. Proudman and Z. UNI Z. 2007. The Effect of Heat Stress on Ovarian Function of Laying Hens. Poult Sci.86:1760-1765.

Sahin, K.; M. Onderci; N. Sahin; MF. Gursi and O. Kucuk. 2003. Dietary Vitamin $\mathrm{C}$ and Folic Acid Supplementation Ameliorates the Detrimental Effects of Heat Stress in Japanese Quail J. Nutr.; 133: 1882 - 1886.

Usman, B. A.; A. U. Mani, A. D. EI - Yuguda and S.S. Diarra. 2008. The Effect of Supplemental Ascorbic Acid on the Development of Newcastle Disease in Japanese Quail (Coturnix coturnix japonica) Exposed to High Ambient Temperature. International Journal of Poultry Science 7 (4): 328-332. 
Weber, G. M. 2009. Improvement of flock productivity through supply of vitamins for higher laying performance and better egg quality. World's Poultry Science Journal, Vol. 65.

Yigit, A. A.; Dikicioglu, T. and Yarim, G. 2002. Effects of increases in vitamin $\mathrm{C}$ supplementation in the laying hen rations on serum concentrations of vitamin $\mathrm{C}$ and vitamin A. Revue Méd. Vét., 153, 8-9, 563-566. 